\title{
A Coupled Electronic and Magnetic Phase Transition in the Infinite-Layer Phase $\mathrm{LaSrNiRuO}_{4}$
}

Midori Amano Patino, Dihao Zeng, Ryan Bower, John E. McGrady, and Michael A. Hayward*

\section{Supporting Information}

Table of Contents

1. Experimental details

Procedure used to measure the magnetization of samples containing elemental nickel.

Figure S1. Plot of magnetisation against field from a $\mathrm{LaSrNiRuO}_{4}$ sample measured at $300 \mathrm{~K}$

2. Chemical characterisation of $\mathrm{LaSrNiRuO}_{4}$

Figure S2. Thermogravimetric data collected while heating $\mathrm{LaSrNiRuO}_{4}$ under a $10 \%$ $\mathrm{H}_{2}$ in $\mathrm{N}_{2}$ atmosphere.

3. Structural refinement of $\mathrm{LaSrNiRuO}_{4}$ against neutron powder diffraction data collected at $300 \mathrm{~K}$.

Table S1. Structural parameters of $\mathrm{LaSrNiRuO}_{4}$ refined against neutron powder diffraction data collected at $300 \mathrm{~K}$ and at $5 \mathrm{~K}$.

Figure S3. Observed calculated and difference plots from the structural refinement of $\mathrm{LaSrNiRuO}_{4}$ against neutron powder diffraction data collected at $300 \mathrm{~K}$.

4. Nuclear and magnetic structural refinement of $\mathrm{LaSrNiRuO}_{4}$ against neutron powder diffraction data collected at $5 \mathrm{~K}$.

Figure S4. Comparison of neutron powder diffraction data collected from $\mathrm{LaSrNiRuO}_{4}$ at $5 \mathrm{~K}$ and $300 \mathrm{~K}$.

Table S2. The magnetic structure of $\mathrm{LaSrNiRuO}_{4}$ refined from neutron powder diffraction data collected at $5 \mathrm{~K}$.

Figure S5. Comparison of fits to neutron diffraction data using three different magnetic models.

Figure S6. Observed calculated and difference plots from the nuclear and magnetic structural refinement of $\mathrm{LaSrNiRuO}_{4}$ against neutron powder diffraction data collected $5 \mathrm{~K}$.

\section{Computational methodology.}

Choice of $U$.

Magnetic coupling.

Figure S7. Three distinct spin configurations of the $\mathrm{LaSrNiRuO}_{4}$ system.

6. References 


\section{Experimental details}

\section{Procedure used to measure the magnetization of samples containing elemental nickel.}

The magnetization of elemental nickel is observed to saturate in applied magnetic fields of more than $2 \mathrm{~T}$. Thus the paramagnetic susceptibility of a bulk sample can be measured in the presence of elemental nickel impurities by measuring the gradient of magnetization-field isotherms in applied fields larger than $2 \mathrm{~T}$. As shown in Figure S1.

To this end the magnetization of samples was measured in a series of 7 fields between $2 \mathrm{~T}$ and $5 \mathrm{~T}$. The magnetization vs. field data were fitted to a linear function, the gradient of which is the paramagnetic susceptibility of the bulk sample and the intercept is the saturated ferromagnetic moment of the sample. Data points with large errors were excluded from fits. All fits had at least 5 data points. This procedure was repeated at $5 \mathrm{~K}$ intervals between $5 \mathrm{~K}$ and $300 \mathrm{~K}$ to measure the temperature dependent susceptibility of samples.

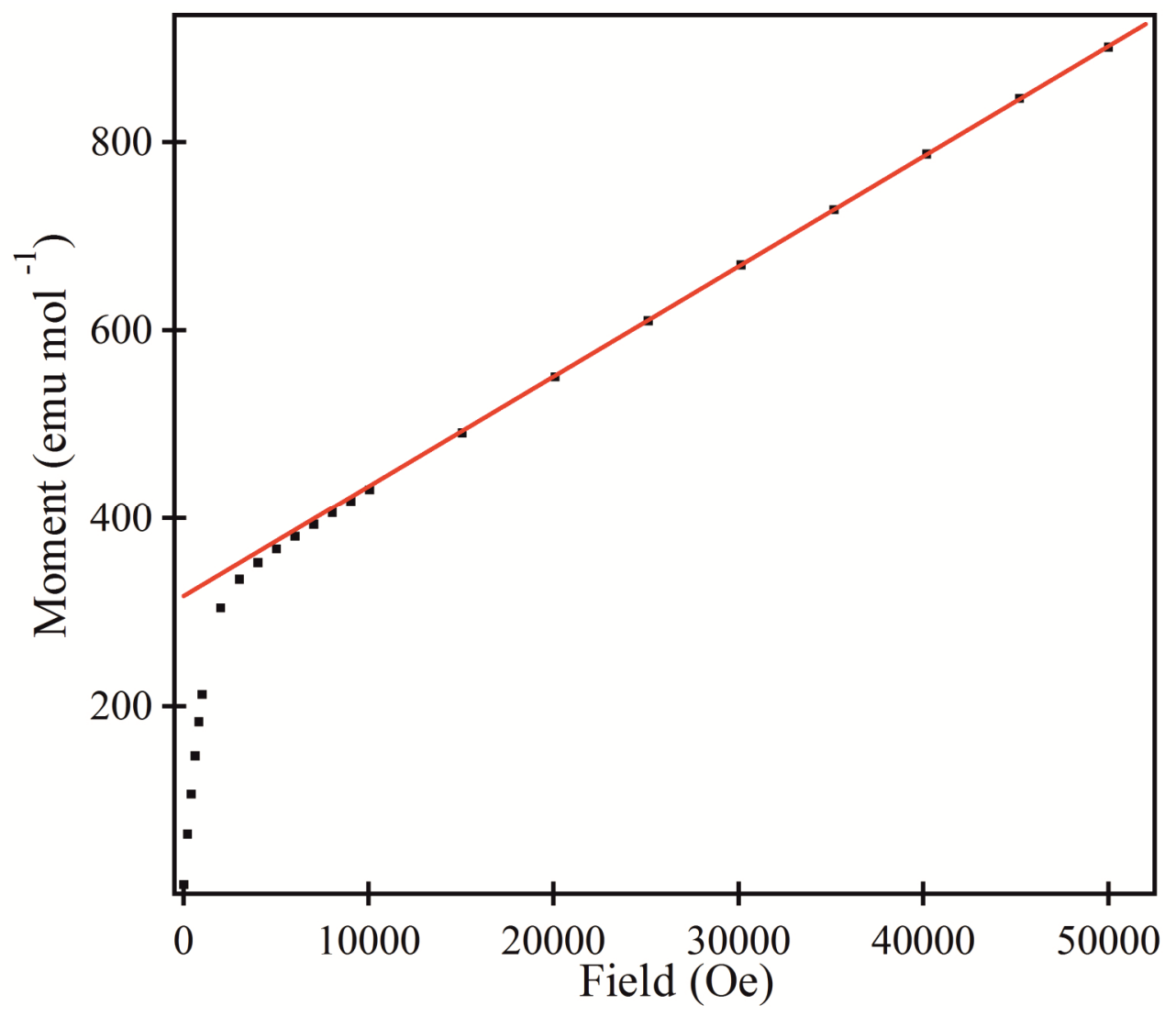

Figure S1. Magnetisation of an $\mathrm{LaSrNiRuO}_{4}$ sample measured as a function of applied field at $300 \mathrm{~K}$. A linear fit to high-field region $(\mathrm{H}>20000 \mathrm{Oe})$ yields a gradient which is the paramagnetic susceptibility of the sample, and an intercept which is the saturated ferromagnetic moment of the sample. 


\section{Chemical characterisation of $\mathrm{LaSrNiRuO}_{4}$}

Thermogravimetric measurements were performed by heating powder samples at a rate of $5 \stackrel{\circ}{\circ} \mathrm{C} \min ^{-1}$ under a $10 \% \mathrm{H}_{2}$ in $\mathrm{N}_{2}$ atmosphere, using a Mettler-Toledo MX1 thermogravimetric microbalance. This procedure lead to the reductive decomposition of the phase to form a mixture of $\mathrm{La}_{2} \mathrm{O}_{3}, \mathrm{SrO}, \mathrm{Ni}$ and $\mathrm{Ru}$, confirmed by powder X-ray diffraction. The mass loss during decomposition (5.38\%, Figure S2) corresponds to an initial composition of $\mathrm{LaSrNiRuO}_{4.01(2) \text {. }}$

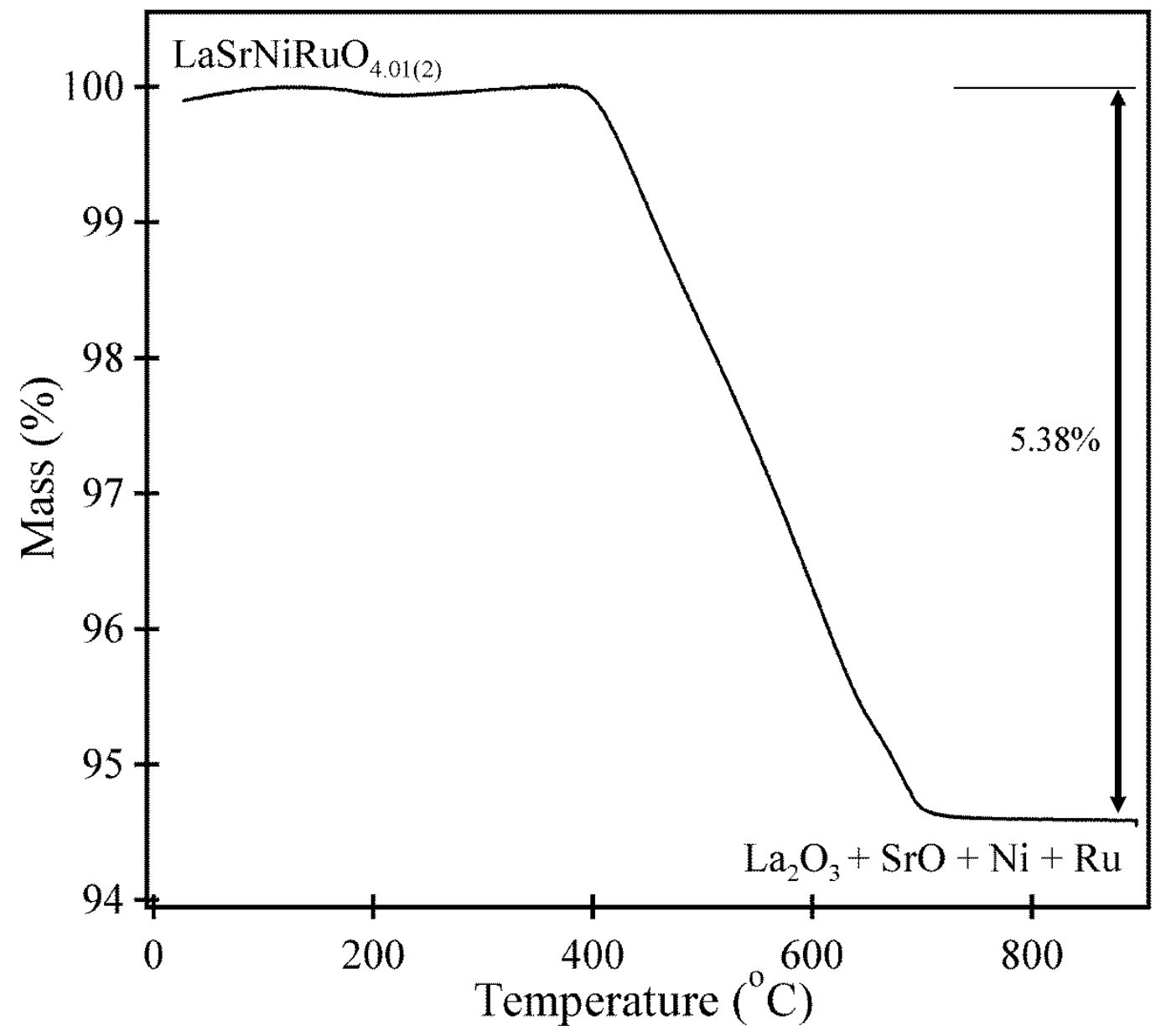

Figure S2. Thermogravimetric data collected while heating $\mathrm{LaSrNiRuO}_{4}$ under a $10 \% \mathrm{H}_{2}$ in $\mathrm{N}_{2}$ atmosphere. 


\section{Structural refinement of $\mathrm{LaSrNiRuO}_{4}$ against neutron powder diffraction data collected at $300 \mathrm{~K}$.}

Neutron powder diffraction data were collected from $\mathrm{LaSrNiRuO}_{4}$ using the POLARIS instrument, at the ISIS neutron source, from samples contained within vanadium cans sealed under argon with indium washers. Rietveld profile refinements were performed using the GSAS suite of programs. ${ }^{[1]}$ The neutron powder diffraction data could be indexed using a body-centred monoclinic unit cell with lattice parameters $a=5.652(3) \AA, b=5.652(3) \AA, c=6.9151(4) \AA, y=90.54(1)^{\circ}$. This unit cell is closely related to that of $\mathrm{LaSrNiRuO}_{6}$ but with a large contraction along the caxis (6.9151(4) $\AA$ vs 7.855(1) $\AA$ ), suggesting that $\mathrm{LaSrNiRuO}_{4}$ adopts an infinite layer structure whilst maintaining the $\mathrm{Ni} / \mathrm{Ru}$ cation order of the $\mathrm{LaSrNiRuO}_{6}$ parent phase. A crystallographic model of this type was constructed in space group $1112 / \mathrm{m}$ and refined against the neutron powder diffraction data. During the refinement all atomic positions were allowed to vary freely and all atoms were described using anisotropic displacement parameters. Close inspection of the data revealed contributions from elemental nickel ( $2.3 \mathrm{wt} \%$ ) as well as $\mathrm{La}_{2} \mathrm{O}_{3}(0.3 \mathrm{wt} \%)$, so these were added to the model as secondary phases. The refinement converged readily to give a good statistical fit. Selected bond lengths and angles are summarized in Table 1 in the main text, with full details of the refined structure in Table S1. Plots of the observed and calculated diffraction data are shown in Figure S3. 


\begin{tabular}{|c|c|c|c|c|c|c|c|c|}
\hline \multicolumn{5}{|c|}{$300 \mathrm{~K}$} & \multicolumn{4}{|c|}{$5 \mathrm{~K}$} \\
\hline Atom & $x$ & $y$ & $z$ & Fraction & $x$ & $y$ & $z$ & Fraction \\
\hline $\mathrm{La} / \mathrm{Sr}$ & 0 & $1 / 2$ & $0.2464(7)$ & $0.5 / 0.5$ & 0 & $1 / 2$ & $0.2450(5)$ & $0.5 / 0.5$ \\
\hline $\mathrm{Ni} / \mathrm{Ru}$ & 0 & 0 & 0 & $0.98(2) / 0.02(2)$ & 0 & 0 & 0 & $0.98(2) / 0.02(2)$ \\
\hline $\mathrm{Ru} / \mathrm{Ni}$ & $1 / 2$ & $1 / 2$ & 0 & $0.98(2) / 0.02(2)$ & $1 / 2$ & $1 / 2$ & 0 & $0.98(2) / 0.02(2)$ \\
\hline $\mathrm{O}(1)$ & $0.251(1)$ & $0.236(1)$ & 0 & 1 & $0.258(1)$ & $0.234(1)$ & 0 & 1 \\
\hline \multirow[t]{2}{*}{$\mathrm{O}(2)$} & $0.245(1)$ & $0.768(1)$ & 0 & 1 & $0.250(1)$ & $0.766(1)$ & 0 & 1 \\
\hline & $\mathrm{U}_{11}$ & $\mathrm{U}_{22}$ & $\mathrm{U}_{33}$ & $\mathrm{U}_{12}$ & $\mathrm{U}_{11}$ & $\mathrm{U}_{22}$ & $\mathrm{U}_{33}$ & $\mathrm{U}_{12}$ \\
\hline $\mathrm{La} / \mathrm{Sr}$ & $0.0048(2)$ & $0.0059(3)$ & $0.0023(1)$ & $0.0016(4)$ & $0.0032(2)$ & $0.0047(2)$ & $0.0020(1)$ & $0.0018(3)$ \\
\hline $\mathrm{Ni}$ & $0.0112(3)$ & $0.0105(2)$ & $0.0098(4)$ & $0.0009(3)$ & $0.0114(2)$ & $0.0101(1)$ & $0.0081(4)$ & $0.0007(3)$ \\
\hline $\mathrm{Ru}$ & $0.0135(3)$ & $0.0148(2)$ & $0.0095(4)$ & $0.0010(4)$ & $0.0142(3)$ & $0.0140(3)$ & $0.0084(4)$ & $0.0012(4)$ \\
\hline $\mathrm{O}(1)$ & $0.0051(1)$ & $0.0068(2)$ & $0.0116(2)$ & $-0.0032(1)$ & $0.0049(1)$ & $0.0048(1)$ & $0.0125(2)$ & $0.0042(1)$ \\
\hline $\mathrm{O}(2)$ & $0.0052(1)$ & $0.0057(1)$ & $0.0118(2)$ & $0.0031(1)$ & $0.0058(1)$ & $0.0057(1)$ & $0.0127(2)$ & $-0.0047(1)$ \\
\hline \multicolumn{5}{|c|}{$\begin{array}{c}\mathrm{LaSrNiRuO}_{4}-\text { Spacegroup I1 } \text { S/m (No.12) } \\
a=5.652(3) \AA, b=5.652(3) \AA, c=6.9151(4) \AA, \\
\gamma=90.54(1)^{\mathrm{o}} \\
\text { volume }=220.89(17) \AA^{3} \\
\text { Weight fraction }=97.4(2) \%\end{array}$} & \multicolumn{4}{|c|}{$\begin{array}{c}\mathrm{LaSrNiRuO}_{4}-\text { Spacegroup } \mathrm{I} \mathrm{S1} / \mathrm{m}(\mathrm{mo.12}) \\
a=5.660(3) \AA, b=5.658(3) \AA, c=6.9007(4) \AA, \\
\gamma=90.70(1)^{\mathrm{o}} \\
\text { volume }=220.97(17) \AA^{3} \\
\text { Weight fraction }=97.4 \%\end{array}$} \\
\hline \multicolumn{5}{|c|}{$\begin{array}{c}\mathrm{Ni}-\text { Spacegroup Fm-3m }(\text { No. } 225) \\
a=3.523(1) \AA \\
\text { Weight fraction }=2.3(3) \%\end{array}$} & \multicolumn{4}{|c|}{$\begin{array}{c}\mathrm{Ni}-\text { Spacegroup Fm-3m }(\text { No. 225) } \\
\begin{array}{c}a=3.520(1) \AA \\
\text { Weight fraction }=2.3 \%\end{array}\end{array}$} \\
\hline \multicolumn{5}{|c|}{$\begin{array}{c}\mathrm{La}_{2} \mathrm{O}_{3}-\text { Spacegroup P-3m1 (No. 164) } \\
a=3.933(1) \AA, c=6.238(1) \AA \\
\text { Weight fraction }=0.3(1) \%\end{array}$} & \multicolumn{4}{|c|}{$\begin{array}{c}\mathrm{La}_{2} \mathrm{O}_{3}-\text { Spacegroup } \mathrm{P}-3 \mathrm{~m} 1(\text { No. } 164) \\
a=3.928(1) \AA, c=6.235(1) \AA \\
\text { Weight fraction }=0.3 \%\end{array}$} \\
\hline \multicolumn{5}{|c|}{$\chi^{2}=5.478, w \mathrm{Rp}=2.13 \%, \mathrm{Rp}=2.19 \%$} & \multicolumn{4}{|c|}{$\chi^{2}=3.199, w \mathrm{Rp}=1.31 \%, \mathrm{Rp}=1.51 \%$} \\
\hline
\end{tabular}

Table S1. Structural parameters of $\mathrm{LaSrNiRuO}_{4}$ refined against neutron powder diffraction data collected at $300 \mathrm{~K}$ and at $5 \mathrm{~K}$. 

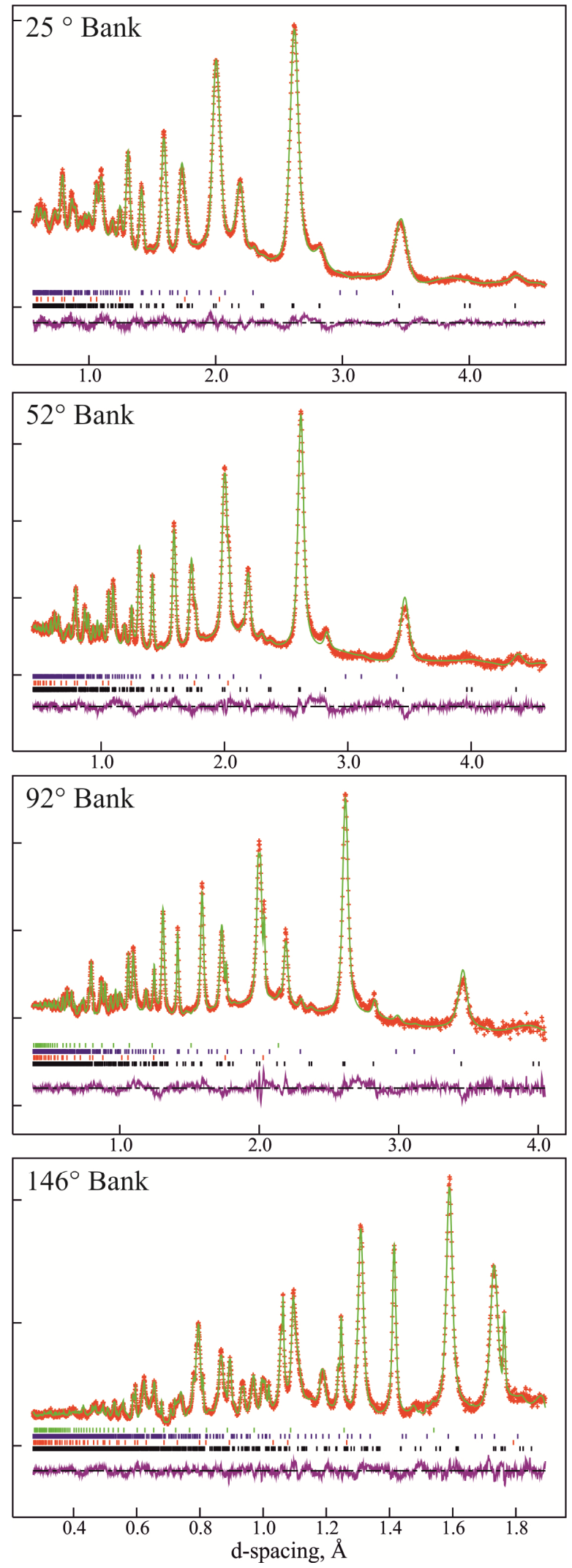

Figure S3. Observed calculated and difference plots from the structural refinement of $\mathrm{LaSrNiRuO}_{4}$ against neutron powder diffraction data collected at $300 \mathrm{~K}$. Black tick marks indicate peak positions for the majority phase; red elemental Ni; purple $\mathrm{La}_{2} \mathrm{O}_{3}$ and green vanadium from the sample holder. 


\section{Nuclear and magnetic structural refinement of $\mathrm{LaSrNiRuO}_{4}$ against neutron powder diffraction data collected at $5 \mathrm{~K}$.}

Comparison of neutron powder diffraction data collected from $\mathrm{LaSrNiRuO}_{4}$ at $300 \mathrm{~K}$ and $5 \mathrm{~K}$ reveals a subtle enhancement in the intensity of some of the diffraction peaks in the low temperature data set (Figure S4), consistent with magnetic order. The magnetic scattering can be indexed using the body-centred crystallographic unit cell, implying that the ordered magnetic lattice is also body centred and therefore that all the nickel centres have the same ordered moment, as do all the ruthenium centres. Magnetic models were constructed on this basis, where the magnetic moments on the nickel and ruthenium centres were allowed to vary independently, and these where refined against the low-temperature diffraction data, in combination with the structural model developed above for the high-temperature diffraction data. The best fit to the data was achieved by aligning both the nickel and ruthenium moments parallel to the z-axis. Under this constraint the model refined to give a moment of 1.03(8) $\mu_{B}$ on the nickel site and $0.05(9) \mu_{B}$ on the ruthenium site, with the two moments aligned parallel. The net moment of 1.08(17) $\mu_{B}$ is in good agreement with the saturated ferromagnetic moment of $\mathrm{LaSrNiRuO}_{4}$ measured at $5 \mathrm{~K}$.

An alternative ferrimagnetic structure in which a moment of $1 \mu_{\mathrm{B}}$ on the nickel centres is anti-aligned with $2 \mu_{\mathrm{B}}$ on the ruthenium sites would also yield a net moment of $\sim 1$ $\mu_{\mathrm{B}}$ per formula unit, however this model gives a very poor fit to the data (Figure S5) and rapidly converts to the ferromagnetic nickel model described above when the magnitude of the ordered moments is refined.

Other alternative models with antiferromagnetic couplings between $\mathrm{Ni}$ or Ru centres (either within the $\mathrm{MO}_{2}$ planes and/or between metal centres in adjacent $\mathrm{MO}_{2}$ planes) have magnetic scattering peaks which are not observed in the experimental data.

Full details of the crystal and magnetic structure of $\mathrm{LaSrNiRuO}_{4}$ refined against the data collected at $5 \mathrm{~K}$ are given in Table $\mathrm{S} 1$ and Table $\mathrm{S} 2$ respectively, with selected bond lengths and angles given in Table 1 in the main text. Plots of the observed and calculated diffraction data are shown in Figure S6. 

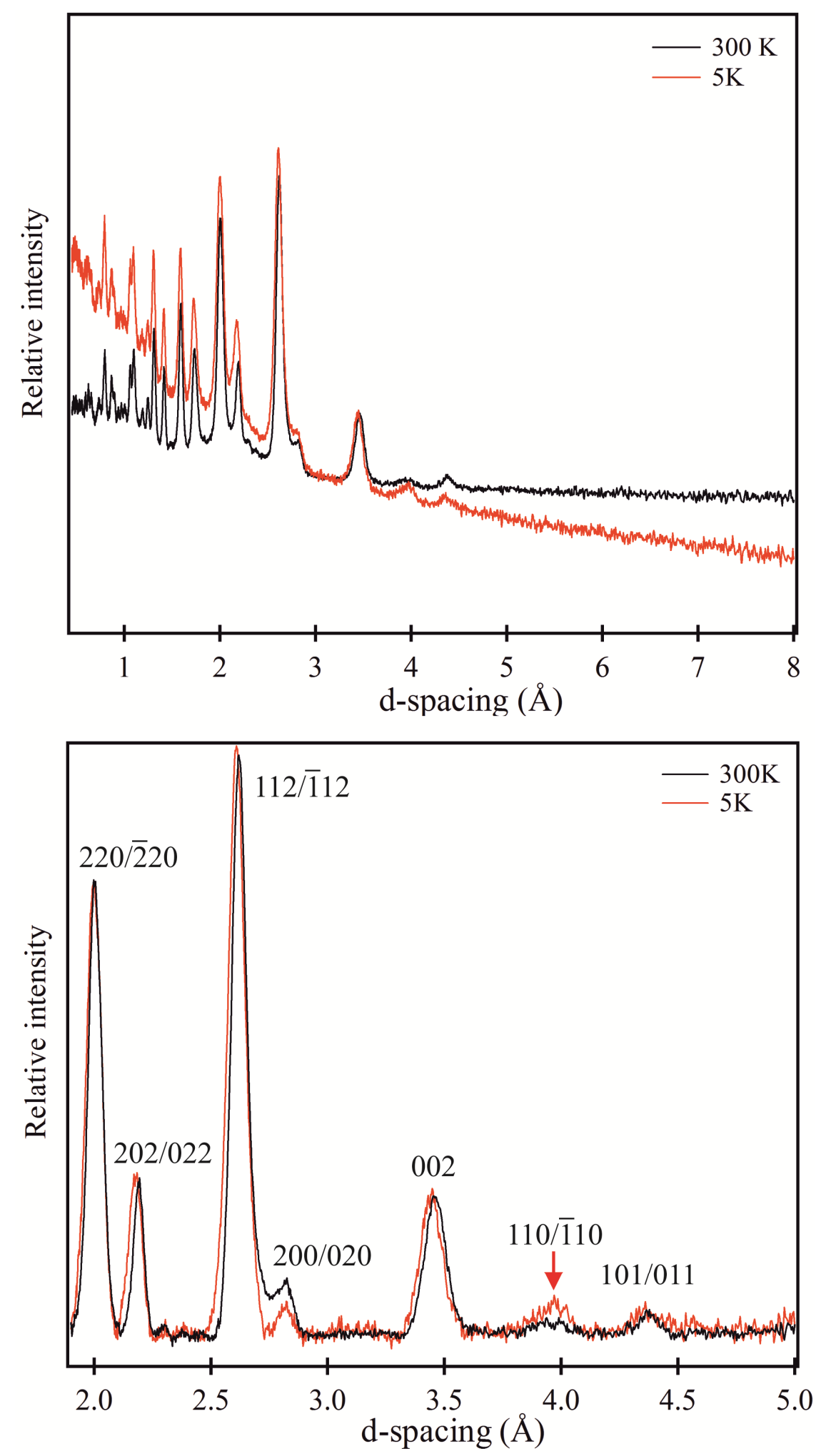

Figure S4. Neutron powder diffraction data collected from $\mathrm{LaSrNiRuO}_{4}$ at $5 \mathrm{~K}$ exhibit enhanced intensity of the 110/-110 diffraction peak, compared to analogous data collected at $300 \mathrm{~K}$, consistent with a magnetically ordered state at low temperature. Top panel shows raw neutron diffraction data, bottom panel shows data after a background subtraction, to highlight the enhancement of the 110/-110 diffraction peak in the $5 \mathrm{~K}$ data set. 


\begin{tabular}{ccccc} 
Atom & $x$ & $y$ & $z$ & $\mathrm{M}_{\mathrm{z}}\left(\mu_{\mathrm{B}}\right)$ \\
\hline $\mathrm{Ni}(1)$ & 0 & 0 & 0 & $1.03(8)$ \\
$\mathrm{Ni}(2)$ & $1 / 2$ & $1 / 2$ & $1 / 2$ & $1.03(8)$ \\
$\operatorname{Ru}(1)$ & $1 / 2$ & $1 / 2$ & 0 & $0.05(9)$ \\
$\operatorname{Ru}(2)$ & 0 & 0 & $1 / 2$ & $0.05(9)$
\end{tabular}

Table S2. The magnetic structure of $\mathrm{LaSrNiRuO}_{4}$ refined from neutron powder diffraction data collected at $5 \mathrm{~K}$. 


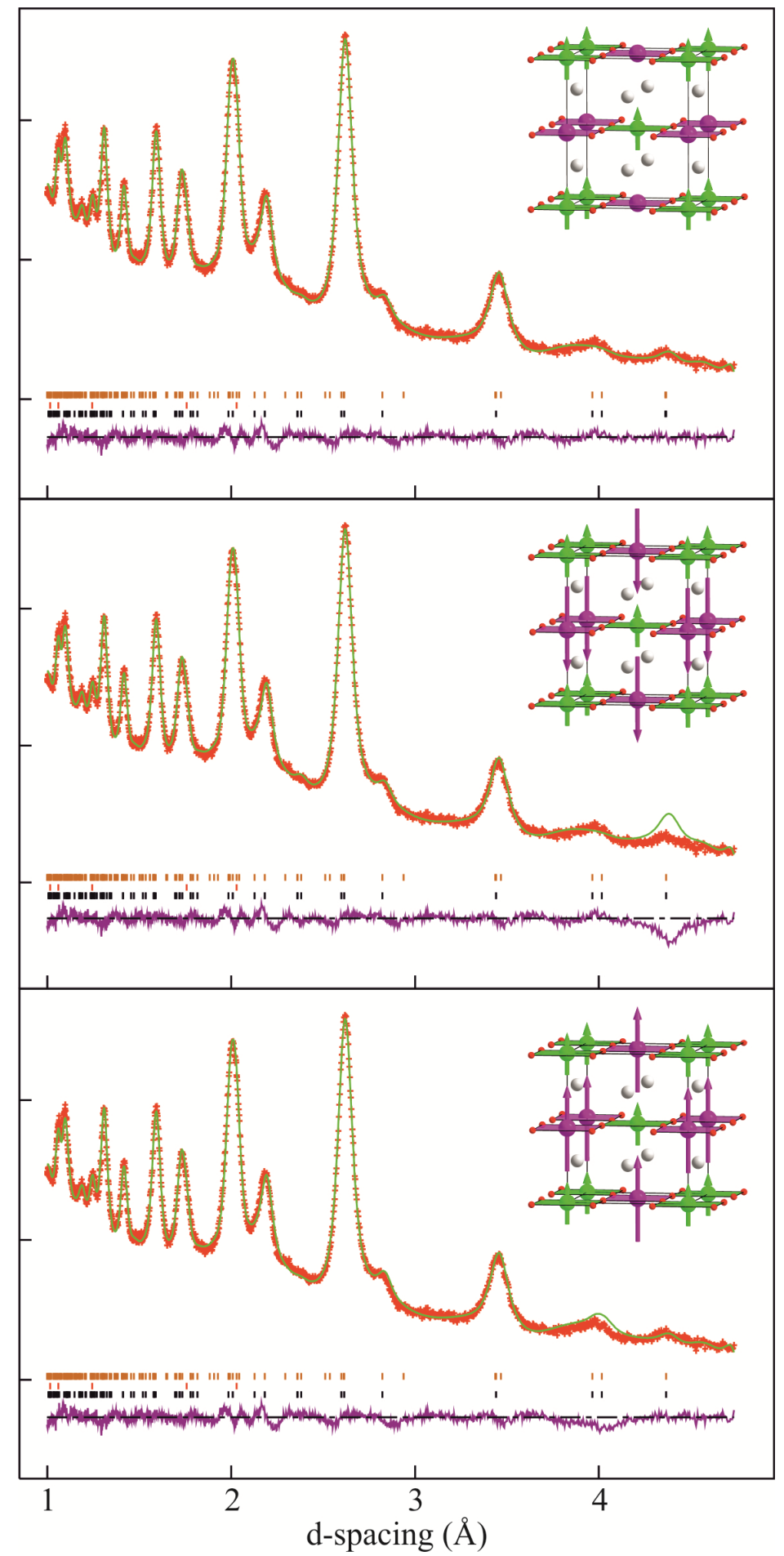

Figure S5. Comparison of the observed calculated and difference plots from the nuclear and magnetic structural refinement of $\mathrm{LaSrNiRuO}_{4}$ against neutron powder diffraction data collected at $5 \mathrm{~K}$, using three different magnetic models.

Top: Ferromagnetic order, $1 \mu \mathrm{B}$ on $\mathrm{Ni}, 0 \mu \mathrm{B}$ on $\mathrm{Ru}$ Middle: Ferrimagnetic order, $1 \mu \mathrm{B}$ on $\mathrm{Ni},-2 \mu \mathrm{B}$ on $\mathrm{Ru}$ Bottom: Ferromagnetic order, $1 \mu \mathrm{B}$ on $\mathrm{Ni}, 2 \mu \mathrm{B}$ on $\mathrm{Ru}$ Black tick marks indicate nuclear peak positions for the majority phase; red elemental $\mathrm{Ni}$; and orange the magnetic cell of $\mathrm{LaSrNiRuO}_{4}$. 

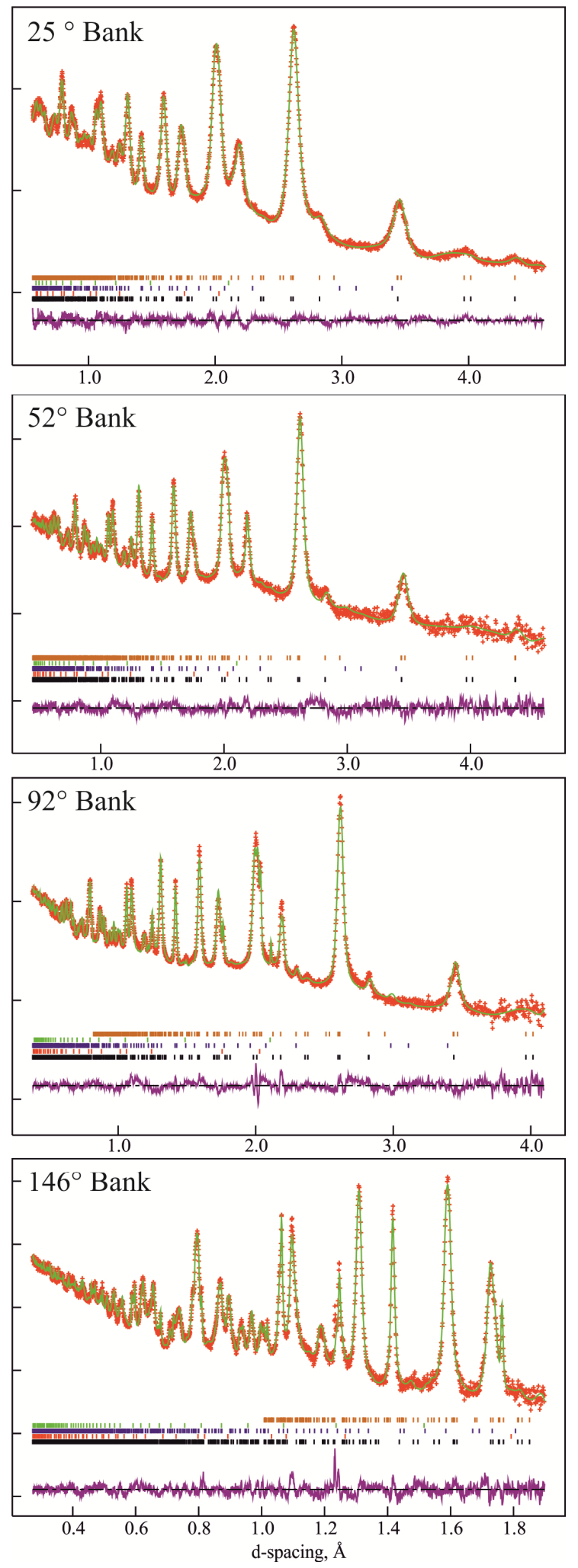

Figure S6. Observed calculated and difference plots from the nuclear and magnetic structural refinement of $\mathrm{LaSrNiRuO}_{4}$ against neutron powder diffraction data collected at $5 \mathrm{~K}$. Black tick marks indicate nuclear peak positions for the majority phase; red elemental $\mathrm{Ni}$; purple $\mathrm{La}_{2} \mathrm{O}_{3}$; green vanadium from the sample holder and orange the magnetic cell of $\mathrm{LaSrNiRuO}_{4}$. 


\section{Computational methodology.}

\section{Further discussion of computational methodology}

\section{(i) Choice of $U$}

Correlation is introduced in these calculations through the addition of $U_{\text {eff }}$ to the Hamiltonian $(\mathrm{DFT}+U)$. The choice of $U_{\text {eff }}$ has been discussed extensively, and is typically larger for the compact $3 d$ orbitals $(\mathrm{Ni})$ than for the more diffuse $4 \mathrm{~d}(\mathrm{Ru})$. Typical values in the literature are $U_{\text {eff }}=6.0 \mathrm{eV}$ for $\mathrm{Ni}$ and $3.0 \mathrm{eV}$ for $\mathrm{Ru}$, and these have been adopted in the calculations reported in the main text. However, given the rather unusual oxidation state prevalent in this low-valent system, $\mathrm{Ni}^{+}$and $\mathrm{Ru}^{2+}$, we have tested the dependence of the results on $U_{\text {eff }}$ across a broad range. The lattice parameters, M-O bond lengths and spin densities prove to be largely independent of the choice of $U_{\text {eff }}$ for $\mathrm{Ni}$ up to $8.0 \mathrm{eV}$. For $\mathrm{Ru}$, in contrast, increasing $U_{\text {eff }}$ increases the spin density at $\mathrm{Ru}\left(\sim 1.50\right.$ for $U_{\text {eff }}=0 \mathrm{eV}$ to $\sim 1.80$ for $U_{\text {eff }}=5 \mathrm{eV}$. At the same time, the lattice angle $\gamma$ is reduced to $\sim 90.5^{\circ}$ and the Ru-O bond lengths become much more similar $(2.00 / 2.04 \AA)$.

\section{Magnetic coupling}

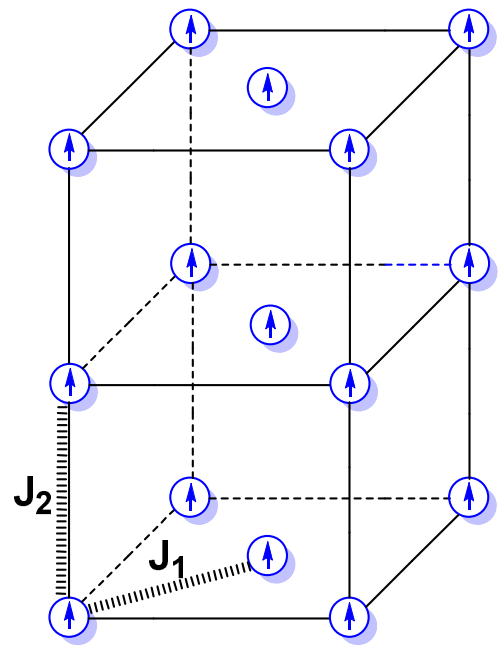

Ferromagnet

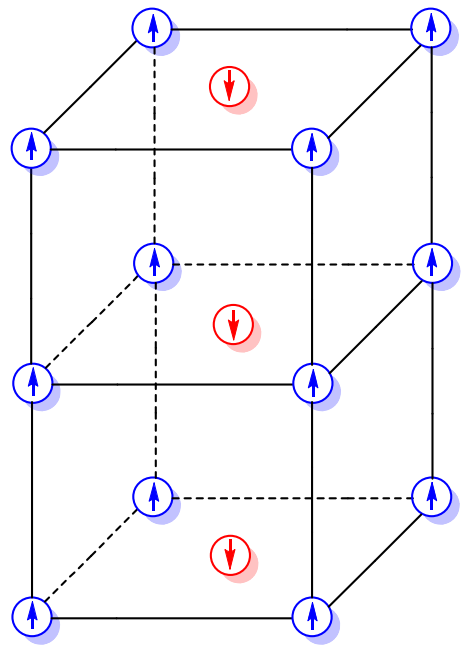

C-type Antiferromagnet

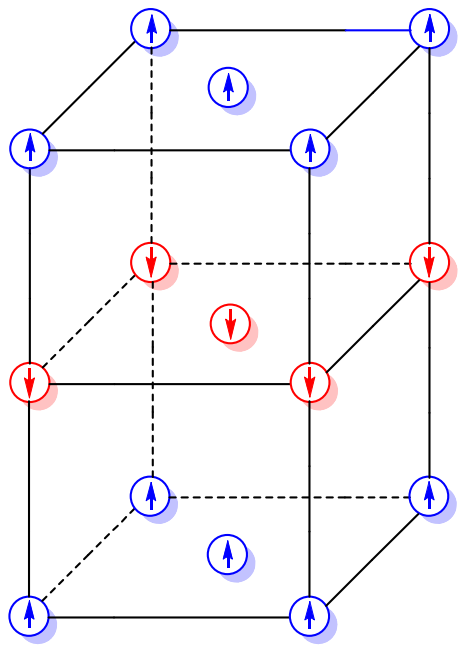

A-type Antiferromagnet

Figure S7. Three distinct spin configurations of the $\mathrm{LaSrNiRuO}_{4}$ system. Circles indicate $\mathrm{Ni} / \mathrm{Ru}$ centres. 
To explore the nature of the magnetic coupling, we have computed the energies of three spin configurations shown in Figure S7, including the all-ferromagnetic state $(\mathrm{F})$, the C-type and A-type antiferromagnets. The energies of these three states are summarised in Table S3: qualitatively it is immediately apparent that the configurations with ferromagnetic coupling in the Ni/Ru planes ( $F$ and $A)$ are substantially more stable than the antiferromagnetic analogue (C).

Table S3. Energies of the three spin configurations

\begin{tabular}{c|c}
$\begin{array}{c}\text { Spin } \\
\text { Configuration }\end{array}$ & Energy $(\mathrm{eV})$ \\
\hline F & -101.187 \\
C & -101.040 \\
A & -101.181
\end{tabular}

These energies can be mapped onto the diagonal elements of the Heisenberg spin Hamiltonian shown below, yielding exchange coupling constants of $J_{1}=1184 \mathrm{~cm}^{-1}$; $J_{2}=46 \mathrm{~cm}^{-1}$.

$$
\widehat{H}=-\sum_{i>j} 2 J_{i j} \hat{s}_{i} \hat{s}_{j}
$$

\section{References}

[1] A. C. Larson, R. B. Von Dreele, Los Alamos National Laboratory Report LAUR 86748, 2000. 